\title{
Cohesin Entangling During Loop Extrusion as a Driving Event for Chromosomal Aberrations: Approach to Modeling BCR-
}

\author{
ABL Translocation
}

\section{Saeed Taheri* \\ The New Lahijan Scientific Foundation, Iran}

*Corresponding author: Saeed Taheri, The New Lahijan Scientific Foundation, Iran, Tel: +98-9121960748; Email: taherimd@gmail.com

Abbreviations: NAHR: Non-Allelic Homologous Recombination.

\section{Introduction}

Chromosomal translocations are usually considered as a hallmark of cancer and they are by far, most prevalent in hematological malignancies. BCR-ABL translocation between chromosomes 9 \& 22 and Philadelphia chromosome is the first translocation ever discovered in the human cells and probably is the most studied one [1]. In this paper, based on the current knowledge by the literature on chromatin structure, its folding as well as their dynamics and characteristics, it is attempted to fabricate mechanistic models for the development of BCRABL translocation.

During the past decade, the concept of chromatin folding has been extensively studied, and they have been implicated in the translocation process in several malignancies [2]. Huge amount of research proposes that chromatin is organized spatially mainly by two competing principle mechanisms:

1) Cohesin-dependent looping which essentially forms at the tag boundaries defined by CCCTC-bindingsequences occupied by CTCF through a mechanism called "loop extrusion model" [2]; and

2) Compartmental higher order organization of the chromatin which is mainly enforced through phase separation [3].
Moreover, it is known that the latter type of looping is able to make contact domains at their anchors even if they reside on different chromosomes, and also their anchors are enriched for super enhancers [4]. It has also been demonstrated that the strength of cohesin-dependent loop formation is adversely associated with the strength of the compartmentalization. Very recently, it has been shown that chromatin at the base of cohesin-dependent loops, called the loop anchors, are very fragile and vulnerable to topoisomerase II mediated double strand breaks (DSBs) in a transcription-, replication-, and celltype-independent manner [2]. On the other hand, we know that translocation sites associated with the BCRABL translocation are mainly defined in specific parts of the chromosomes $9 \& 22$ (i.e. m-bcr, M-bcr, $\mu$-bcr in chromosome $22 \& \mathrm{ABL}$ breakpoint regions at the exons 1a-2) both ubiquitously transcribed genes. On the other hand, it has been shown that breakage points within genic/intergenic regions (both happening in the process of BCR-ABL translocation [5]) are highly enriched at the range of active enhancers demarcated by H3K27ac+ histone marks [2]. So, if we speculate that the breakage sites associated with the BCR-ABL translocation do actually reside at the loop anchors (though no suggestive evidence exists yet in the literature), the explanation will not be a very complex one.

\section{Competing Forces Promote the Translocation}

The main idea here is that, an external (or internal) stimulus exerts stress to the chromatin in either chromosomes 9 or 22 and the most fragile sites at the 
loop anchors would be broken apart. This disintegration of chromatin structure due to the DSB formation gives more opportunity to the rival forces including compartmentalization and super enhancers to redistribute the intra- \& inter-chromosomal folding and configurations. It is already known that the two chromosomes $9 \& 22$ are in very close proximity in the hematological lineage cell nucleus. This fact, together with our knowledge on the highly concentrated active histone mark (H3K27ac+) at either the topological loop domain anchors [2] and/or the induction of $\gamma$-H2AX histone marks on the breakage sites of both chromosomes, and all in conjunction with the help from the diffusion forces might be able to bring the breakage sites together and promote the translocation (Figure 1). However this model doesn't explain why the particular domains associated with BCR \& ABL genes are that much prone to translocation than several other loop domains on the same or other chromosomes. The survival preference offered by the BCR-ABL translocation cannot make a good explanation since most cell with the translocation won't survive for long, and most people representing it never develop the disease. So, a more sophisticated model is needed.

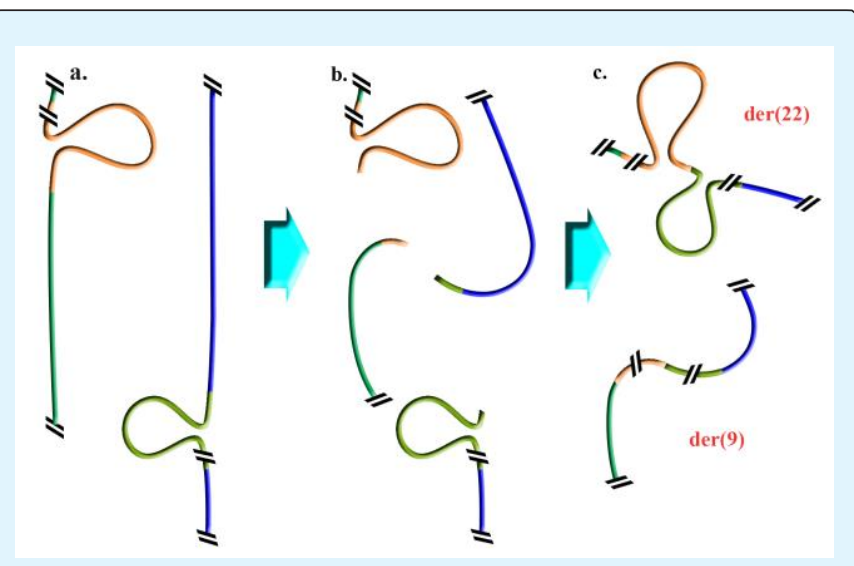

Figure 1: BCR-ABL translocation mechanism based on the "competing forces model"; a. Chromosomes 9 (blue; right) \& 22 (turquoise; left) in close proximity in the nuclear space; $b$. The stimuli induces double strand breaks at the loop anchor sites of ABL gene (green) and BCR (orange). Compartmentalization and diffusion forces bring the DSB sites on either chromosomes in close contact; c. chromosomal rearrangement between chromosomes $9 \& 22$ at the breakage sites, producing BCR-ABL gene fusion on der (22) (Philadelphia chromosome), and ABL-BCR on der (9).

\section{Non-Allelic Homologous Recombination (NAHR)}

Saglio, et al. [5] in their study of a patient with CML and Philadelphia chromosome reported existence of a 76 $\mathrm{Kb}$ duplicon region in both chromosomes 9 \& 22, about $150 \mathrm{~Kb} 3^{\prime}$ to the BCR and $1.4 \mathrm{Mb} 5$ ' to ABL genes. They proposed that this region somehow plays a role in the translocation but no mechanism that explains its role had been presented. Nevertheless if we can determine mechanism of DSBs happening in either chromosome, the role of NAHR in the translocation process could be more readily defined as an inter-chromosomal anchor point that would bring the two chromosomal breakage sites to a vicinity, making a distinct explanation why these special sections of the two genes are that much prone to translocation than their neighboring sequences, or why similar translocations don't occur for every two chromosomes that might be in spatial nuclear proximity to each other (Figure 2).

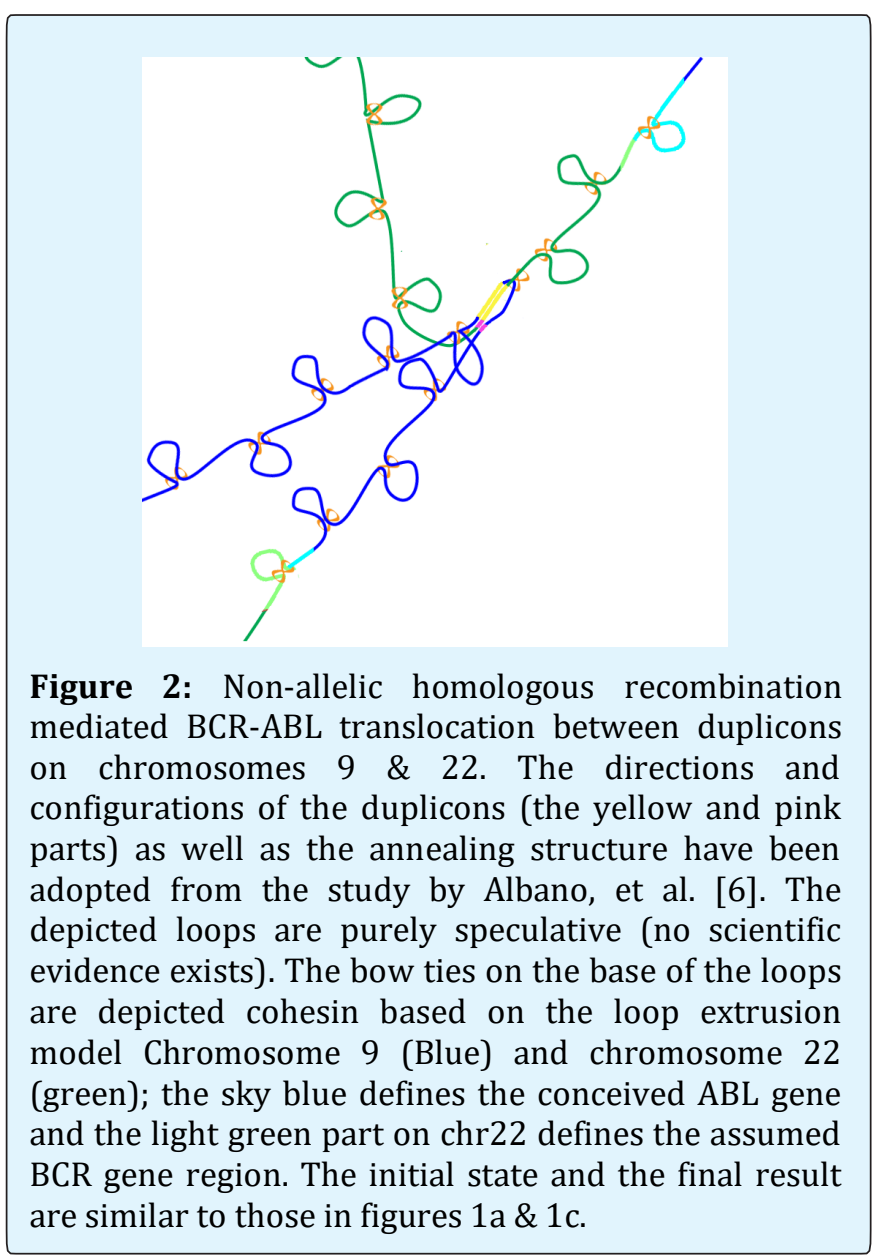


NAHR may also provide an unprecedented and splendid explanation for a model in which there is no need for simultaneous DSB formation in both the chromosomes and in which the second breakpoint can be not only be happening subsequent to the first one, but also as a consequence of it. The scenario here is that after the first DSB happening in the ABL conventional breakage site and subsequent annealing of the duplicons in chromosomes $9 \& 22$, and because the duplicon on chromosome 22 is in relatively short distance to the BCR gene, the latter event could exert strains on the BCR gene, which is supposed to make matrix/scaffold attachment regions on its attachment points to the nuclear surface (supposedly on its major rearrangement sites $\mathrm{m}$-bcr \& Mbcr) and induce disruption of the chromatin (Figure 3). Then after, the two chromatins may join together at their breakpoints. Although too much speculative, one may even think that even if the first breakpoint is somewhere else than on the ABL gene, the annealing of the two chromosomes may make some unknown conformations and interactions to chromosome 9 that finally leads to breakage at the exact $\mathrm{ABL}$ consensus conventional rearrangement sites (see below).

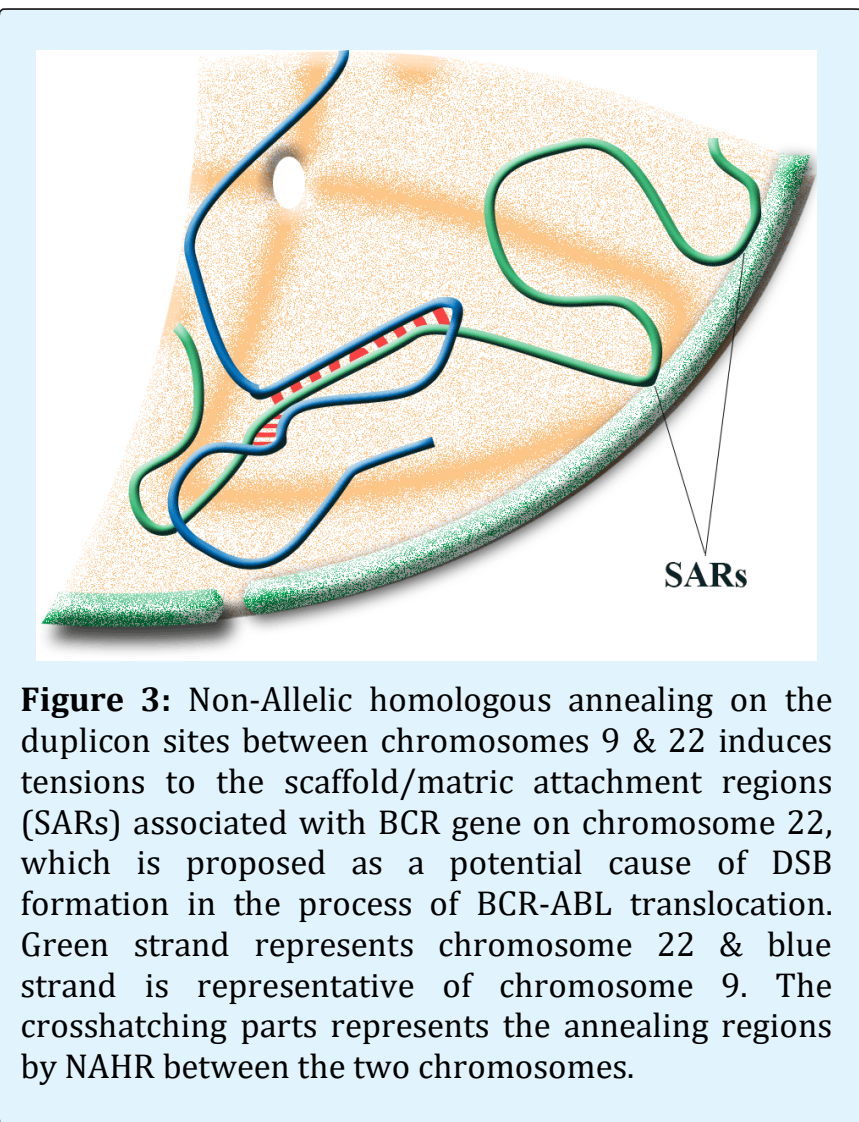

Saeed Taheri. Cohesin Entangling During Loop Extrusion as a Driving Event for Chromosomal Aberrations: Approach to Modeling BCR-ABL Translocation. Cytol Histol Int J 2019, 3(1): 000110.

\section{DSBs Dislocation/Juxtaposition}

One possibility for the augmented detection of BCR$\mathrm{ABL}$ translocation might be related to the disintegration of the breakage sites on either chromosomes subsequent to the NAHR formation and then juxtaposition of the DSB sites of the opposite chromosomes together. Figure 4 depicts this idea.

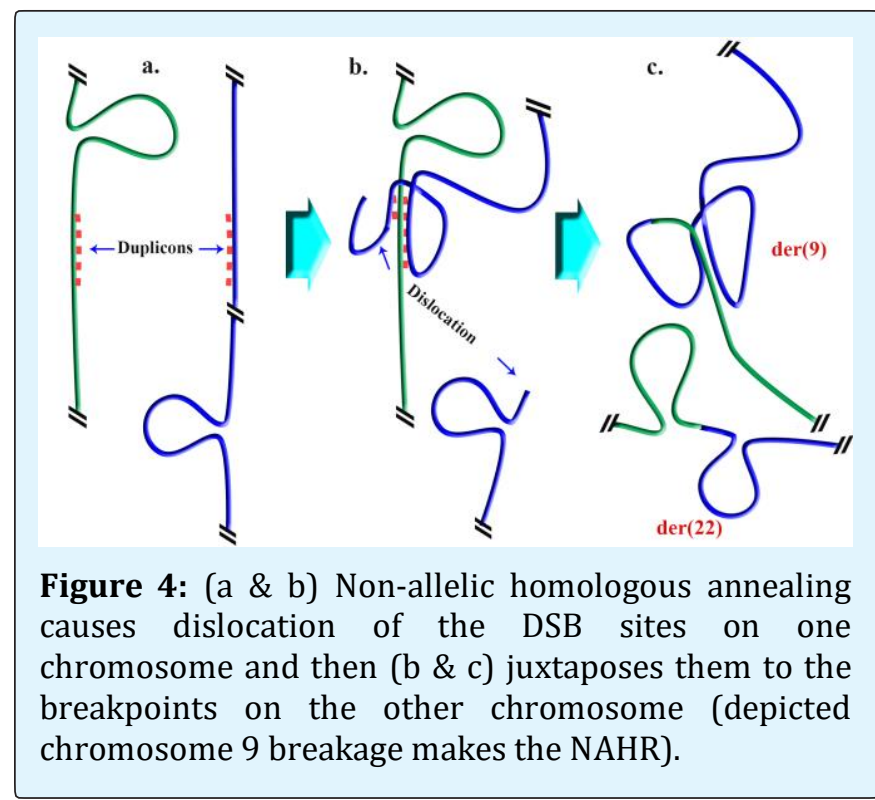

\section{Loop Extrusion Entanglement}

Loop extrusion model is very well described in reference 4 . The above mentioned idea that proposes breakpoints at either BCR or ABL genes exist at the loop anchors is a weak idea for at least two reasons: 1. No topological loop domains have yet been reported at either $\mathrm{BCR}$ or $\mathrm{ABL}$ breakpoints in Hi-C studies; and 2. The range within which the breaks happen in either gene are much larger than the range we expect from the loop anchor boundaries. In fact it is highly probable that nonpublished studies have already investigated this idea finding none; because this had been proposed for some other leukemia translocations where actually loop domains had been responsible [2]. Nevertheless, there still could be some hypothetical explanations that might provide models with no need to speculate the breakage sites residing on the loop domain boundaries. First, it has been shown that topological loop domains with CTCFcohesin binding at their basements are not the only loops developing in the chromatin and enhancer-promoter loops, loops made by superenhancers or at compartment domains peaks, are among other types of loops made in 
the chromatin $[2,3]$. On the other hand, it has been shown that the level of relationship between Hi-C-defined loop anchors and DSBs was greater than the overlap between loop anchors and CTCF/RAD21 co-occupancy [2], which means that not only it is the chromatin loop itself, and not any specific type of it that predicts DSB formation but also, CTCF-cohesin at the loop anchors might be more protective than eliciting to DSB formation. Moreover, data is distinctive that cohesin is the single most powerful predictive of DSB occurrence to the extent that, RAD21 detection on the chromatin alone has been suggested as the single best predictive of DSB formation [2].

According to the loop extrusion model, cohesin after loading to the chromatin slides in opposite directions in both tethered chromatin stands until getting stopped by reaching bound CTCF proteins. And when the cohesin reaches the CTCF bound on one side, sliding continues on the other strand until getting stopped by a well oriented
CTCF and make a loop. Nevertheless, we can assume that, after cohesin getting to a CTCF bound site on one of the tethered chromatin arms, the other tethered cohesin might be instead paused by some other type of chromatin anchors, including the abovementioned ones, making a loop with a cohesin located on the basement (Figure 5). And since it is well known that the loops that frequently harbor DSBs are the ones with increased interactions [4], if the second anchor point is a super enhancer hub, or enhancer-promoter loop or compartment domain, all offering amplified interactions, this promiscuous anchoring of a cohesin extrusion - which is proposed to company topoisomerase II enzyme during its extrusion traveling - will be an ideal point for DSB formation (Figure 5). And in fact, the second break of the translocation might actually occur through this process, and due to the new interactions made through intra- \& interchromosomal intermingling.

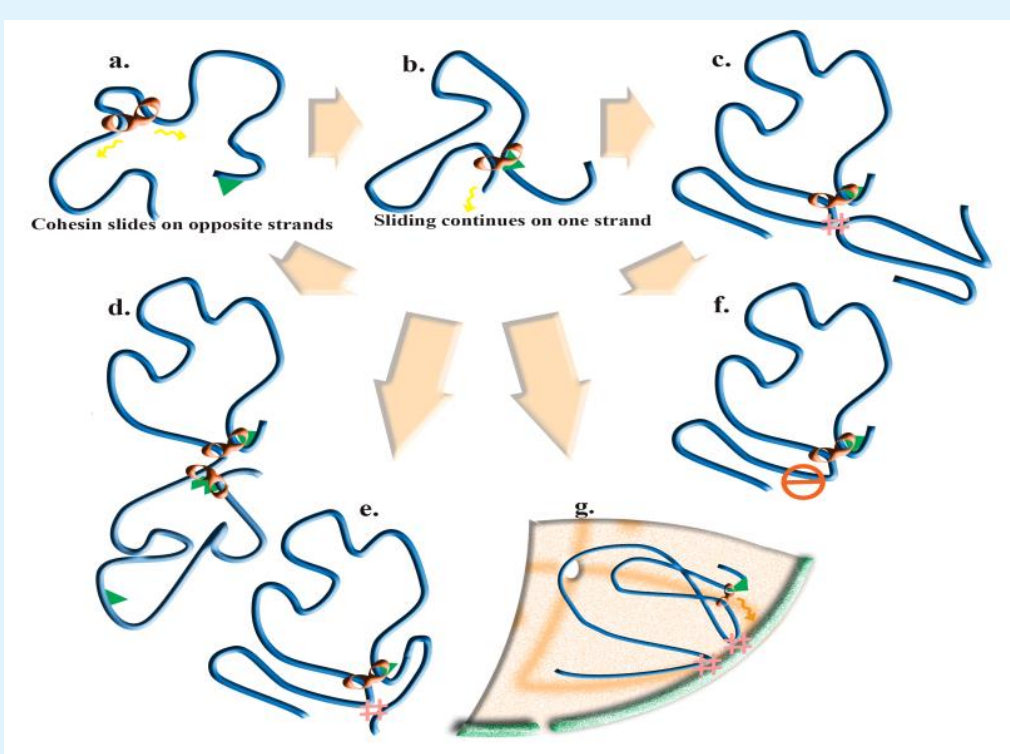

Figure 5: Depiction of the possible illicit scenarios happening during loop extrusion model (a well description of which can be found in reference 4) that may lead to double strand breaks (DSB); a. cohesin starts extrusion on both strands on opposite directions (a well oriented CTCF site is depicted on one of the strands); b. cohesin is locked up on one strand after reaching the CTCF site but it continues sliding on the other stand; c. cohesin sliding on the second strand is stopped due to reaching an interlink between two chromatin strands which can be on one or two different chromosomes (a good representative for an enhancer-promoter loop); d. the second strand sliding gets stopped after reaching the anchor base to another loop; e. cohesin sliding the second strand gets stopped due to reaching a link between the two strand of the same chromatin (it can be simply a larger loop of any type); f. cohesin sliding is stopped in the second strand due to reaching to a bulky element on the chromatin, preventing it from further sliding; g. cohesin sliding at on the second strand gets stopped after reaching the matrix attachment region of the chromatin. Notice: In the figures, it is speculated that the cohesin gets docked, at least on one strand, by a well oriented CTCF. But it is quite possible that the entangling occurs on both strands. 
Five major reasons can be provided for the increased fragility induced by the promiscuous entangling of the sliding cohesin: 1. the anchor-point is associated with 3 or more strands linked together (vs. conventional 2 strands); 2. The anchor-point is associated with chromatin bending; 3. It also predicts anchor-points between two different chromosomes; 4. Inflexibility (anchor-points at the CTCF is a flexible one because it is predicted that the sliding cohesin can escape one or more CTCF sites, but in no case can it escape intra- and inter-chromosomal links); and 5. The strain caused by tensions made by two competing interactions (i.e. like loop extrusion and nuclear matrix attachment regions (Figures $3 \& 5$ ). And finally in most of the abovementioned interactions, there are a combination of these increased fragility factors imposed on the chromatin fiber(s).

\section{Conclusion}

This study was a step by step attempt to model BCRABL translocation. Prior to this study, some attempts had been made in this regard [6,7]; however in this study, author tried to employ the most recent data presented to the literature, in order to model the translocation in a way that it can provide a more precise mechanistic explanation to its different features \& characteristics. In brief, an insult to the one of the chromosomes (presumably chromosome 9 \& maybe on ABL gene) promotes annealing/NAHR, and this event causes significant tensions and disturbances to the normal chromosomal states in either chromosomes which can make breakages in either of them in their conventional rearrangement sites potentially by different mechanisms (Figures $3 \& 5$ ) and then also annealing/NAHR dislocates the DSB sites on either chromosomes and prevents a rapid repair by any physiological mechanisms and then juxtaposes them to the breakage sites on the other chromosome (Figure 4). The final results are the rearranged chromosomes der (22) containing BCR-ABL translocation and the reciprocal ABL-BCR on der (9). So, the current modeling provides some explanations for the variability of the rearrangement points in either chromosomes as well as the specific range within which these rearrangements occur. Moreover, it provides an interesting explanations for the high frequency of the BCR-ABL translocation detected in different settings by proposing more a lower repair rate for the breakages than the conventional idea of higher fragility. In fact, the idea of a causative role for the "loop extrusion entanglement" by factors other than the CTCF boundaries probably able to explain much more of the chromosomal aberrancies observed in different settings. But on the other hand, the distinctive role of NAHR versus just annealing between the homologous sequences has not been well defined in a way that it can explain which one may play the essential role in the process; moreover, in the modeling steps presented here, presumptions have been made based on only indirect evidence, and therefore extensive research would be needed to confirm the modeling presented by this commentary or to rule it out.

\section{References}

1. Goldman JM, Melo JV (2003) Chronic myeloid leukemia--advances in biology and new approaches to treatment. N Engl J Med 349(15): 1451-1464.

2. Canela A, Maman Y, Jung S, Wong N, Callen E, et al. (2017) Genome Organization Drives Chromosome Fragility. Cell 170(3): 507-521.e18.

3. Tiwari VK, McGarvey KM, Licchesi JD, Ohm JE, Herman JG, et al. (2008) PcG proteins, DNA methylation, and gene repression by chromatin looping. PLoS Biol 6(12): 2911-2927.

4. Rao SSP, Huang SC, Glenn St Hilaire B, Engreitz JM, et al. (2017) Cohesin Loss Eliminates All Loop Domains. Cell 171(2): 305-320,e24.

5. Saglio G, Storlazzi CT, Giugliano E, Surace C, Anelli L, et al. (2002) A 76-kb duplicon maps close to the BCR gene on chromosome 22 and the $\mathrm{ABL}$ gene on chromosome 9: possible involvement in the genesis of the Philadelphia chromosome translocation. Proc Natl Acad Sci U S A 99(15): 9882-9887.

6. Albano F, Anelli L, Zagaria A, Coccaro N, D'Addabbo P, et al. (2010) Genomic segmental duplications on the basis of the $t(9 ; 22)$ rearrangement in chronic myeloid leukemia. Oncogene 29(17): 2509-2516.

7. González García JR, Cruz MD, Gutiérrez CB (2015) Are submicroscopic chromosomal inversions predisposing factors for the $t(9 ; 22)(q 34 ; q 11.2)$ translocation in chronic myeloid leukemia?. Mol Cytogenet 8: 14. 